\title{
Synthesis, antibacterial and antioxidant activity of novel 12-(N-arylmethaniminyl)indolo[1,2-c]quinazolines
}

\author{
Aditya DIXIT 1* (D) , Devender PATHAK ${ }^{2}$ (D) , Gyanendra Kumar SHARMA ${ }^{1}$ (D) \\ 1 Rajiv Academy for Pharmacy, Mathura, U.P., India \\ 2 Pharmacy College, Saifai, Etawah, U.P., India \\ * Corresponding Author. E-mail: adixit70@gmail.com (A.D.); Tel. +919258474535.
}

Received: 05 October 2018 / Revised:08 December 2018 / Accepted: 10 December 2018

\begin{abstract}
The objective of the present research was to synthesize 12 -( $N$-arylmethaniminyl)indolo [1,2-c]quinazoline derivatives (4a1-4a11) and further characterize in terms of ${ }^{1} \mathrm{H}$ NMR, ${ }^{13} \mathrm{C}$ NMR, FTIR and mass spectrophotometry. Evaluation of their antibacterial and antioxidant activity was also carried out. Most compounds have shown a good antioxidant activity in all the concentrations but compounds 4a7, 4a8 and $4 \mathrm{a} 11$ were found to be most potent antioxidants in comparison to others with an $\mathrm{IC}_{50}$ value of $18.78 \pm 1.86 \mu \mathrm{g} / \mathrm{ml}, 16.84 \pm 2.60 \mu \mathrm{g} / \mathrm{ml}$ and 18.64 $\pm 2.40 \mu \mathrm{g} / \mathrm{ml}$, respectively (DPPH method) and $18.83 \pm 2.89 \mu \mathrm{g} / \mathrm{ml}, 16.61 \pm 3.00 \mu \mathrm{g} / \mathrm{ml} \& 16.8 \pm 3.34 \mu \mathrm{g} / \mathrm{ml}$, respectively $\left(\mathrm{H}_{2} \mathrm{O}_{2}\right.$ method). Antibacterial activity was done against $B$. subtilis and $E$. coli with the help of agar well diffusion method and it showed that all the derivatives in the series are having good antibacterial activity but compounds $4 \mathbf{a 9}$ and 4 a11 were found to be most promising antibacterial agents. It was concluded that the compounds containing substituted anilines (4-nitro, 4-fluoro, 4-bromo and 4-chloro-2-nitro) on the methaniminyl group of 12th position of indoloquinazoline moiety have good antioxidant and antibacterial activities.
\end{abstract}

KEYWORDS: Synthesis; indoloquinazoline; antioxidant; antibacterial.

\section{INTRODUCTION}

If we talk about indole, then it is the most trusted component of the chemical constituents present in alkaloids [1,2]. It consists of diverse pharmacological roles as well, for example antiviral activity [3-6], anticancer activity [7, 8], antimicrobial activities [9, 10], antitubercular activitiy [11], antioxidant activity and antifungal activity [12]. Antimicrobial and antioxidant activities are the most prominent activities of indole nucleus. Many chemical structures containing indole have been reported by the pharmaceutical chemists as antimicrobial and antifungal compounds. From all those structures, ethyl-3-indolylacrylate, 5-bromo-3-(2-cyanovinyl)indole and 3(2-nitrovinyl)indole were those derivatives which were effective against varieties of microorganisms [13]. A concentration between $10-100 \mu \mathrm{g} / \mathrm{ml}$ was the highly effective in case of haloindoles. One research study illustrated that some 3-Acyl-4,7-dihydroxy indoles were found to be active against Escherichia coli and Streptococcus pyogenes [14]. 1-Morpholino-3-carbethoxy-5-hydroxy- 2-methylindole was found to be most potent derivative against Escherichia coli and Bacillus cirroflagellosus [15]. 1-(4-Phenyl) and (1-naphthyl-4H-1,2,4-triazole-5-thion-3-yl)indoles were reported as strong antibacterial and antifungal compounds [16]. Some thiosemicarbazide compounds containing indole moiety and their cyclic 1,2,4-triazole and 1,3,4-thiadiazole derivatives were reported to be selective against microbes and fungi [17]. Several derivatives of indole fused with different heterocycles were reported and their antimicrobial potential was also determined. Out of them, $4 H$-pyrano[2,3-f]indole, benzotetrahydrocyclohept[1,2-b]indole, and 1-triazolylethylbenz $[g]$ indole derivatives were found to be most active [18-20]. On the other hand, indole is a promising moiety for free radical scavenging activity. Many evidences are there about the involvement of free radicals in a variety of diseases and pathophysiological events

How to cite this article: Dixit A, Pathak D, Sharma G.K. Synthesis, antibacterial and antioxidant activity of novel 12-( $N$-arylmethaniminyl) indolo[1,2c]quinazolines. J Res Pharm. 2019; 23(3): 584-595. 
including inflammation, cancer, myocardial infarction, arthritis neurodegenerative disorders [21-23]. This serious involvement of free radical action on vital systems is a multiple complex aspect of their involvement in a series of inflammatory diseases [24] and diseases related to nutrition $[25,26]$. There are so many anti-inflammatory agents which act through scavenging of free oxygen radicals $[27,28]$. There is production of large amount of free radicals during the process of inflammation, out of a wide range of radicals, reactive oxygen species (ROS) such as, superoxide radical $\left(\mathrm{O}_{2}{ }^{-}\right)$, hydrogen peroxide $\left(\mathrm{H}_{2} \mathrm{O}_{2}\right)$, hypochlorous acid $(\mathrm{HOCl})$, singlet oxygen and peroxyl radical, as well as reactive nitrogen species (RNS), like nitric oxide (NO) and peroxynitrite anion (ONOO-). Indeed, ROS and RNS are produced by the endothelial cells, Kupffer cells, neutrophils and macrophages as mechanism of defense in response to infections caused by foreign pathogens [29-32]. On the other hand, ROS are also produced in the process of inflammation as a consequence of COX enzyme activity. Mitochondria are also considered as a great source of ROS [33]. These ROS and RNS are attached with a wide variety of pathologies and disorders such as cancer, rheumatoid arthritis, atherosclerosis, Alzheimer and Parkinson disease, among others [34]. In way of exploring some more potential of indole moiety, synthesis of some novel Indolo[1,2-c]quinazoline derivatives (4a1-4a11) was carried out and their antibacterial and antioxidant activity was reported.

\section{RESULTS AND DISCUSSION}

\subsection{Chemistry}

In the starting of scheme, phenyl hydrazone of 2-Aminoacetophenone was synthesized by refluxing equimolar quantities of phenyl hydrazine and 2-Aminoacetophenone in acetic acid/ethanol mixture for $3 \mathrm{~h}$. Then, 2-(2-aminophenyl)indole (1a) was synthesized by cyclization of phenylhydrazone of 2-Aminoacetophenone in the presence of phosphorous pentoxide and methanesulphonic acid. Further cyclization of $1 \mathbf{a}$ in presence of $90 \%$ Formic acid gave Indolo[1,2-c]quinazoline (2a). Compound 2a was allowed to undergo formylation by VilsmeyerHaack Formylation to yield 12-formylindoloquinazoline (3a). Compound 3a reacted with substituted anilines to give 12-( $N$-arylmethaniminyl)indolo[1,2-c]quinazolines (4a1-4a11). As shown by Billimoria et al reaction scheme for the compounds 1a, 2a and 3a was utilized which is presented below (Figure 1) [35].

The characterization of compounds was done with the help of IR, ${ }^{1} \mathrm{H}$ NMR, ${ }^{13} \mathrm{C}$ NMR, mass spectral data, and elemental analysis. In the IR spectrum of compounds 4a1-4a11, the aromatic C-H stretching vibrations gave rise to a band at 3080-3019 $\mathrm{cm}^{-1}$. Stretching bands for aliphatic C-H were observed in the region of $2958-2798 \mathrm{~cm}^{-}$ 1. The $\mathrm{C}-\mathrm{Cl}$ stretching bands were observed at $752-692 \mathrm{~cm}^{-1}$. The stretching vibrations of nitro group gave rise to bands at 1554-1508 $\mathrm{cm}^{-1}$ and 1360-1308 $\mathrm{cm}^{-1}$. Stretching vibrations of C-F and C-Br were observed at $1292 \mathrm{~cm}^{-1}$ and $551 \mathrm{~cm}^{-1}$ respectively.

In the ${ }^{1} \mathrm{H}$ NMR spectrum of compound 4a1-4a11, the signals due to the protons of the aromatic rings appeared between 7.00 and $9.23 \mathrm{ppm}$ in the form of singlet, doublet, double doublet and multiplet. The signal due to the $\mathrm{C}-\mathrm{H}$ proton of methaniminyl group was observed at $7.50 \mathrm{ppm}$ as a singlet. Spectra of compounds showed the signal due to the proton of quinazoline ring at $9.23 \mathrm{ppm}$ as a singlet.

In the ${ }^{13} \mathrm{C}$ NMR spectra of all compounds, signals due to carbons present in aromatic rings were observed in the region 102-160.1 ppm. The signal due to the $\mathrm{N}-\mathrm{C}=\mathrm{N}$ carbon of quinazoline ring was observed at $136 \mathrm{ppm}$. The signal due to the carbon of $\mathrm{C}-\mathrm{Cl}$ was observed at 127-135 ppm. The signal due to the C-F carbon appeared at $161.40 \mathrm{ppm}$. The signal due to the $\mathrm{C}-\mathrm{Br}$ carbon appeared at $121.63 \mathrm{ppm}$. Mass spectral data and elemental analysis were complying with the structures of compounds. [M+2] peaks were found for compounds with $\mathrm{Cl}$ moiety.

\subsection{Biological activities}

All the newly synthesized indole derivatives (4a1-4a11) were screened for their antibacterial and antioxidant activity. 


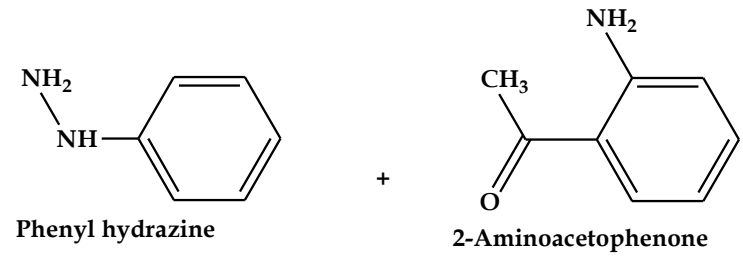

\begin{tabular}{c|c}
$\mathrm{AcOH} / \mathrm{EtOH}$ \\
Reflux
\end{tabular} \mid $\begin{gathered}\mathrm{P}_{2} \mathrm{O}_{5} / \mathrm{CH}_{3} \mathrm{SO}_{3} \mathrm{H} \\
80-100^{\circ} \mathrm{C}\end{gathered}$

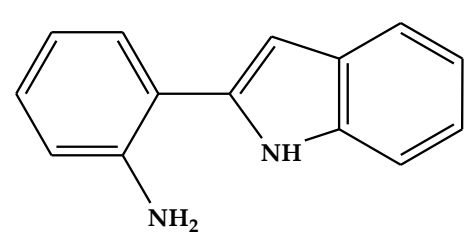

2-(2-aminophenyl)indole

1a

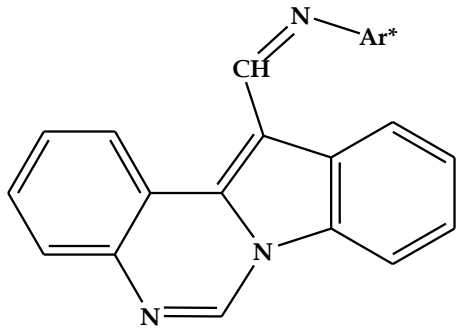

12-( $N$ - arylmethaniminyl)indoloquinazoline

4a1-4a11

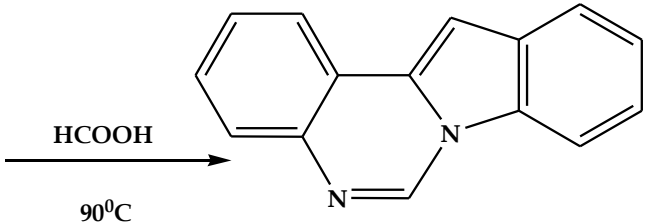

Indoloquinazoline

2a

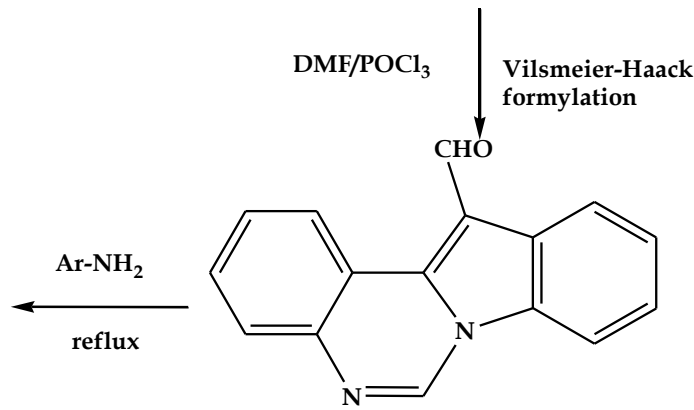

12-formylindoloquinazoline

$3 a$

Figure 1. Synthetic route to 12-(N-arylmethaniminyl)indolo[1,2-c]quinazolines.

*Ar: Phenyl, 2-Chlorophenyl, 3-Chlorophenyl, 4-Chlorophenyl, 2-Nitrophenyl, 3-Nitrophenyl, 4-Nitrophenyl, 4-Fluorophenyl, 4-Bromophenyl, 2-Chloro-4-nitrophenyl, 4-Chloro-2-nitrophenyl.

\subsubsection{Antibacterial activity}

Synthesized compounds were not shown significant antibacterial activity at the concentration range of 10 to $30 \mathrm{\mu g} / \mathrm{ml}$. So, the higher concentrations of compounds were used to evaluate antibacterial efficacy. As shown in observations (Table 1), compound $\mathbf{4 a 9}$ showed $11 \mathrm{~mm}$ and $18 \mathrm{~mm}$ zone of inhibition at $100 \mu \mathrm{g} / \mathrm{ml}$ and 150 $\mu \mathrm{g} / \mathrm{ml}$ concentrations, respectively against $B$. subtilis. Compound $4 \mathbf{4 1 1}$ showed maximum antibacterial activity against E.coli at $100 \mu \mathrm{g} / \mathrm{ml}$ concentration (zone of inhibition $10 \mathrm{~mm}$ ). Ciprofloxacin showed better antimicrobial activity against $B$. subtilis and E. coli at lower concentration $(25 \mu \mathrm{g} / \mathrm{ml})$. 
Table 1. Zone of inhibition values for derivatives (4a1-4a11) at $100 \mu \mathrm{g} / \mathrm{ml}$ and $150 \mu \mathrm{g} / \mathrm{ml}$.

\begin{tabular}{cccccc}
\hline Compounds & \multicolumn{5}{c}{ Zone of inhibition (mm) } \\
\hline & \multicolumn{3}{c}{ B. subtilis } & \multicolumn{3}{c}{ E.coli } \\
\cline { 2 - 5 } & $\mathbf{1 0 0} \mathbf{\mu g} / \mathbf{m l}$ & $\mathbf{1 5 0} \mathbf{\mu g} / \mathbf{m l}$ & $\mathbf{1 0 0} \mathbf{\mu g} / \mathbf{m l}$ & $\mathbf{1 5 0} \mathbf{~ g} / \mathbf{m l}$ \\
\hline $\mathbf{4 a 1}$ & 0 & 5 & 5 & 7 \\
$\mathbf{4 a 2}$ & 3 & 9 & 8 & 10 \\
$\mathbf{4 a 3}$ & 2 & 10 & 6 & 11 \\
$\mathbf{4 a 4}$ & 4 & 9 & 6 & 12 \\
$\mathbf{4 a 5}$ & 2 & 6 & 11 & 10 \\
$\mathbf{4 a 6}$ & 10 & 13 & 8 & 15 \\
$\mathbf{4 a 7}$ & 4 & 10 & 9 & 13 \\
$\mathbf{4 a 8}$ & 6 & 12 & 10 & 15 \\
$\mathbf{4 a 9}$ & 11 & 18 & 11 & 16 \\
$\mathbf{4 a 1 0}$ & 8 & 14 & 10 & 17 \\
$\mathbf{4 a 1 1}$ & 9 & 16 & & 10 \\
\hline
\end{tabular}

\subsubsection{Antioxidant activities}

\section{DPPH radical scavenging activity}

The newly synthesized compounds were screened for free radical scavenging activity by DPPH method. The samples were prepared at concentrations of 10,20,30, 40 and $50 \mu \mathrm{g} / \mathrm{ml}$, and ascorbic acid was taken as standard. Compounds 4a7, 4a8 and $\mathbf{4 a 1 1}$ were found to have good scavenging activity with an $\mathrm{IC}_{50}$ value of 18.78 $\pm 1.86 \mu \mathrm{g} / \mathrm{ml}, 16.84 \pm 2.60 \mu \mathrm{g} / \mathrm{ml}$ and $18.64 \pm 2.40 \mu \mathrm{g} / \mathrm{ml}$, respectively. The tabular representation of percentage of free radical scavenging activity is shown (Table 2).

$\mathrm{H}_{2} \mathrm{O}_{2}$ radical scavenging activity

Compounds were screened for free radical scavenging activity by $\mathrm{H}_{2} \mathrm{O}_{2}$ method. The samples were prepared at concentrations of 10, 20,30, 40 and $50 \mu \mathrm{g} / \mathrm{ml}$, and ascorbic acid was taken as standard. Compounds 4a7, 4a8 and 4a11 were found to have good scavenging activity with an $\mathrm{IC}_{50}$ value of $\quad 18.83 \pm 2.89 \mu \mathrm{g} / \mathrm{ml}$, $16.61 \pm 3.00 \mu \mathrm{g} / \mathrm{ml}$ and $16.8 \pm 3.34 \mu \mathrm{g} / \mathrm{ml}$, respectively. The tabular representation of percentage of free radical scavenging activity is shown (Table 3 ).

\subsection{Structure activity relationship}

Above stated results showed that all those compounds which were having para substitutions, they were found to be potent antibacterials and antioxidants. Compounds 4a9 (4-Bromophenyl substituent) and 4a11 (4Chloro-2-Nitrophenyl substituent) were found to be most promising antibacterials among all other synthesized compounds. Compounds 4a7 (4-nitrophenyl substituent), 4a8 (4-fluorophenyl substituent) and 4a11 (4-chloro-2nitrophenyl substituent) were found to be most potent antioxidants due to the presence of electron withdrawing groups at para position. Another electron withdrawing group at ortho position can enhance the antioxidant activity, it can be clearly observed in case of compound $4 \mathbf{a 1 1}$ where nitro group is attached at ortho position along with chlorine atom at para position. 
Table 2. DPPH free radical scavenging activity of compounds 4a1-4a11.

\begin{tabular}{cccccc}
\hline Compounds & \multicolumn{5}{c}{ \% Inhibition at $\mathbf{\mu g} / \mathbf{m l}^{*}$} \\
\hline & $\mathbf{1 0}$ & $\mathbf{2 0}$ & $\mathbf{3 0}$ & $\mathbf{4 0}$ & $\mathbf{5 0}$ \\
\hline Ascorbic acid & $45.8 \pm 1.21$ & $54.01 \pm 1.09$ & $61.60 \pm 0.98$ & $66.89 \pm 1.23$ & $78.01 \pm 2.31$ \\
$\mathbf{4 a 1}$ & $04.21 \pm 1.01$ & $14.15 \pm 0.95$ & $24.69 \pm 1.13$ & $32.22 \pm 1.15$ & $39.45 \pm 2.01$ \\
$\mathbf{4 a 2}$ & $21.68 \pm 1.10$ & $29.21 \pm 1.03$ & $37.65 \pm 1.22$ & $39.45 \pm 1.45$ & $42.16 \pm 2.32$ \\
$\mathbf{4 a 3}$ & $17.16 \pm 1.50$ & $21.38 \pm 1.16$ & $26.20 \pm 1.21$ & $30.42 \pm 1.33$ & $36.74 \pm 1.91$ \\
$\mathbf{4 a 4}$ & $12.95 \pm 1.41$ & $20.18 \pm 1.20$ & $26.80 \pm 1.27$ & $31.02 \pm 1.29$ & $34.33 \pm 1.80$ \\
$\mathbf{4 a 5}$ & $09.03 \pm 1.53$ & $18.07 \pm 1.62$ & $24.69 \pm 0.81$ & $34.03 \pm 1.16$ & $45.78 \pm 1.09$ \\
$\mathbf{4 a 6}$ & $06.62 \pm 1.21$ & $12.95 \pm 1.15$ & $18.97 \pm 0.99$ & $23.49 \pm 1.87$ & $27.40 \pm 2.03$ \\
$\mathbf{4 a 7}$ & $45.83 \pm 1.12$ & $50.32 \pm 1.30$ & $54.48 \pm 1.51$ & $64.74 \pm 1.03$ & $71.47 \pm 1.99$ \\
$\mathbf{4 a 8}$ & $47.28 \pm 1.31$ & $51.50 \pm 1.02$ & $55.12 \pm 1.56$ & $61.44 \pm 1.28$ & $66.56 \pm 1.27$ \\
$\mathbf{4 a 9}$ & $16.86 \pm 1.18$ & $24.09 \pm 1.26$ & $27.40 \pm 1.40$ & $31.32 \pm 1.29$ & $35.24 \pm 1.33$ \\
$\mathbf{4 a 1 0}$ & $29.21 \pm 1.52$ & $31.92 \pm 1.01$ & $33.73 \pm 1.25$ & $36.74 \pm 1.36$ & $40.06 \pm 1.40$ \\
$\mathbf{4 a 1 1}$ & $45.48 \pm 1.14$ & $51.50 \pm 1.26$ & $55.12 \pm 1.71$ & $60.54 \pm 1.21$ & $67.46 \pm 2.06$ \\
\hline
\end{tabular}

${ }^{*} \mathrm{n}=3$ (results are average of triplicate readings with standard deviation)

Table 3. $\mathrm{H}_{2} \mathrm{O}_{2}$ free radical scavenging activity of compounds 4a1-4a11.

\begin{tabular}{cccccc}
\hline Compounds & \multicolumn{5}{c}{ \% Inhibition at $\mathbf{\mu g} / \mathbf{m l} \mathbf{l}^{*}$} \\
\hline & $\mathbf{1 0}$ & $\mathbf{2 0}$ & $\mathbf{3 0}$ & $\mathbf{4 0}$ & $\mathbf{5 0}$ \\
\hline Ascorbic acid & $44.35 \pm 1.21$ & $55.32 \pm 1.34$ & $62.09 \pm 1.51$ & $66.72 \pm 1.10$ & $77.02 \pm 1.33$ \\
$\mathbf{4 a 1}$ & $07.34 \pm 1.14$ & $17.10 \pm 1.61$ & $21.22 \pm 1.24$ & $35.43 \pm 1.91$ & $43.45 \pm 1.99$ \\
$\mathbf{4 a 2}$ & $20.41 \pm 1.05$ & $33.35 \pm 2.01$ & $42.56 \pm 1.14$ & $46.87 \pm 1.82$ & $55.96 \pm 1.90$ \\
$\mathbf{4 a 3}$ & $16.32 \pm 1.76$ & $24.55 \pm 2.21$ & $31.48 \pm 1.88$ & $41.09 \pm 1.67$ & $47.21 \pm 1.62$ \\
$\mathbf{4 a 4}$ & $20.76 \pm 1.55$ & $27.86 \pm 1.91$ & $32.99 \pm 1.77$ & $38.58 \pm 1.24$ & $44.67 \pm 1.85$ \\
$\mathbf{4 a 5}$ & $10.31 \pm 1.36$ & $22.84 \pm 1.78$ & $28.45 \pm 1.82$ & $37.50 \pm 1.08$ & $47.08 \pm 1.19$ \\
$\mathbf{4 a 6}$ & $03.23 \pm 1.47$ & $13.65 \pm 1.39$ & $20.88 \pm 2.22$ & $31.78 \pm 1.29$ & $36.66 \pm 1.02$ \\
$\mathbf{4 a 7}$ & $44.60 \pm 2.01$ & $51.25 \pm 1.98$ & $55.03 \pm 1.96$ & $65.62 \pm 1.21$ & $71.09 \pm 0.92$ \\
$\mathbf{4 a 8}$ & $46.65 \pm 1.58$ & $52.15 \pm 1.21$ & $56.23 \pm 1.73$ & $61.78 \pm 1.56$ & $67.34 \pm 0.86$ \\
$\mathbf{4 a 9}$ & $14.32 \pm 1.25$ & $22.49 \pm 2.02$ & $29.77 \pm 2.05$ & $33.54 \pm 1.69$ & $39.06 \pm 1.41$ \\
$\mathbf{4 a 1 0}$ & $28.21 \pm 1.05$ & $37.22 \pm 1.99$ & $41.42 \pm 1.59$ & $45.65 \pm 2.13$ & $50.62 \pm 2.20$ \\
$\mathbf{4 a 1 1}$ & $46.17 \pm 2.09$ & $52.45 \pm 1.68$ & $56.87 \pm 1.12$ & $61.22 \pm 1.52$ & $68.33 \pm 1.28$ \\
\hline
\end{tabular}

${ }^{*} \mathrm{n}=3$ (results are average of triplicate readings with standard deviation)

\section{CONCLUSION}

Synthesis of some new 12-( $N$ - arylmethaniminyl)indolo[1,2-c]quinazolines (4a1-4a11) was carried out and then they were characterized by the ${ }^{1} \mathrm{H}$ NMR, ${ }^{13} \mathrm{C}$ NMR, FTIR and mass spectrophotometry. They were evaluated for their potential as an antioxidant and an antibacterial. Most of them have shown good antioxidant potential in all the dilutions. Compounds 4a7, 4a8 and 4a11 were found to be most potent antioxidants. Antibacterial studies showed that the compounds $\mathbf{4 a 9}$ and $\mathbf{4 a 1 1}$ have exhibited good spectrum of antibacterial activity. With these results, it can be concluded that the compounds containing para substituted anilines (4-nitro, 4-fluoro, 4-bromo \& 4-chloro-2-nitro) on the methaniminyl group of $12^{\text {th }}$ position of indoloquinazoline moiety have good antioxidant and antibacterial activities. In future, proposed compounds may be analyzed using in- vivo model for better results. 


\section{MATERIALS AND METHODS}

\subsection{Chemistry}

Purity determination of all the derivatives was done by TLC on pre-coated silica gel aluminum sheets (Type 60 GF254, Merck) and iodine vapors and UV-Lamp were used as detecting agents of spots. The melting point determination was done by melting point apparatus and all the melting points were uncorrected. The infrared (FT-IR) spectra were recorded on 470- Shimadzu FTIR spectrophotometer and all values of wavenumber were expressed in $\mathrm{cm}^{-1}$. NMR spectra were measured in DMSO- $d 6$ as solvent at $300 \mathrm{MHz}\left({ }^{1} \mathrm{H} \mathrm{NMR}\right)$ and $75 \mathrm{MHz}\left({ }^{13} \mathrm{C}\right.$ NMR) on a BRUKER AVANCE-300 spectrometer using tetramethylsilane (TMS) as an internal standard. Chemical shifts $(\delta)$ are given in parts per million (ppm). Spin multiplicities are given as s (singlet), d (doublet), dd (double doublet), $\mathrm{t}$ (triplet) and $\mathrm{m}$ (multiplet). Mass spectra were obtained on Shimadzu 2010A LC-MS spectrometer. Elemental analysis was done on Elemental Vario EL III Carlo Erba 1108 and the values were within $\pm 0.04 \%$ of the theoretical values.

\section{Experimental procedure for the synthesis of 2-(2-Aminophenyl)indole (1a)}

Phenyl hydrazone of 2-Aminoacetophenone was synthesized by refluxing equimolar quantities of phenyl hydrazine and 2-Aminoacetophenone in acetic acid/ethanol mixture for $3 \mathrm{~h}$. Methanesulfonic acid (225 ml) was heated to $80{ }^{\circ} \mathrm{C}$ and phosphorus pentoxide $(31.5 \mathrm{~g})$ was added slowly to it with stirring until it dissolved completely (mixture A). The phenylhydrazone of 2-Aminoacetophenone (22.5 g) was slowly added to mixture A, maintaining the temperature between 80 and $100{ }^{\circ} \mathrm{C}$. The solution was then further heated at $80{ }^{\circ} \mathrm{C}$ for 30 min. The reaction mixture was then cooled to room temperature and poured over crushed ice containing sodium hydroxide. The solid precipitate was filtered, washed with water, and dried to afford the crude product which was crystallized from ethanol.

(Yield: 18.77 g, $83.42 \%$, M.p. $144-145^{\circ} \mathrm{C}$ )

Experimental procedure for the synthesis of Indolo[1,2-c]quinazoline (2a)

In a beaker, 2-(o-Aminopheny1)indole $(18 \mathrm{~g})$ was added to formic acid $(88 \%, 135 \mathrm{ml})$ and the solution was heated at $90{ }^{\circ} \mathrm{C}$ for $1 \mathrm{~h}$. The reaction mixture was then cooled to room temperature and poured over crushed ice. The solid precipitate was filtered, washed with water, and dried to afford crude product which was crystallized from ethanol.

(Yield: 13.67 g, $72.48 \%$, M.p. $202-203{ }^{\circ} \mathrm{C}$ )

Procedure for the synthesis of 12-Formylindolo[1,2-c]quinazoline (3a)

Vilsmeyer-Haack formylation

Phosphorous oxychloride (10.66 g) was slowly added to $N, N$-Dimethylformamide (410 ml)

at $0{ }^{\circ} \mathrm{C}$ and the solution was stirred for $15 \mathrm{~min}$ and was added drop wise to Indolo[1,2-c]quinazoline (13.67 g) and the mixture was stirred for a further $15 \mathrm{~min}$ and then refluxed for $30 \mathrm{~min}$. It was then cooled to room temperature and poured over crushed ice. The precipitate was filtered, washed with water $(3 \times 100 \mathrm{ml})$ and boiled with aqueous sodium hydroxide $(5 \%, 100 \mathrm{ml})$. The solid was then filtered, washed with water till free of alkali, and dried. The crude material was crystallized from methanol to give compound $3 \mathbf{a}$.

(13.21 g, $84.95 \%$, M.p. $\left.230-232{ }^{\circ} \mathrm{C}\right)$

General procedure for the synthesis of 12-(N-Arylmethaniminyl)indolo[1,2-c]quinazolines (4a1-4a11)

A mixture of 12-Formylindoloquinazoline $(1 \mathrm{~g}, 4.06 \mathrm{mmol})$ and anilines $(4.06 \mathrm{mmol})$ in methanol was refluxed for $1 \mathrm{~h}$. After cooling the mixture was poured on ice to give precipitate. The product was filtered, washed with cold water and recrystallized with ethanol. Percentage yield was found in between 80-90 \%.

$N-((H-i n d o l o[1,2-c] q u i n a z o l i n-12-y l) m e t h y l e n e) b e n z e n a m i n e ~(4 a 1)$

Compound 3a $(1 \mathrm{~g})$ was refluxed with Aniline $(0.37 \mathrm{~g})$.

(Yield: 1.17 g, 90\%); M.p. $250-251{ }^{\circ} \mathrm{C}$; 
FT-IR ( $\left.v_{\max }, \mathrm{cm}^{-1}\right) 3060$ (Aromatic C-H str.), 2798 (Aliphatic C-H str.), 1614 (C=N str.), 1559, 1525 (Aromatic $\mathrm{C}=\mathrm{C}$ ring str.), 1350 (Aromatic $\mathrm{C}-\mathrm{N}$ str.);

${ }^{1} \mathrm{H}$ NMR (300 MHz, DMSO-d6) $\delta$ ppm: $7.05(\mathrm{dd}, 1 \mathrm{H}), 7.15(\mathrm{dd}, 1 \mathrm{H}), 7.32(\mathrm{~s}, 5 \mathrm{H}), 7.42(\mathrm{~d}, 1 \mathrm{H}), 7.50(\mathrm{~s}, 1 \mathrm{H}), 7.58(\mathrm{dd}$, $1 \mathrm{H}), 7.67(\mathrm{~d}, 1 \mathrm{H}), 7.78(\mathrm{dd}, 1 \mathrm{H}), 7 . .84(\mathrm{~d}, 1 \mathrm{H}), 8.06(\mathrm{~d}, 1 \mathrm{H}), 9.25(\mathrm{~s}, 1 \mathrm{H}) ;{ }^{13} \mathrm{C}$ NMR $(75 \mathrm{MHz}, \mathrm{DMSO}-d 6) \delta$ ppm: 102.01, $111.13,119.21,120.11,122.20,122.39,122.50,125.42,126.14,127.32,127.41,127.83,128.52,130.10,130.24,134.34$, 136.62, 144.24, 149.12, 149.41, 155.45, 160.21;

EIMS (m/z): [M]+ 321.36, [M+1]+ 322.37; Fragments: 217.20, 189.37, 163.22, 108.23, 27.14; Anal. calcd. for $\mathrm{C}_{22} \mathrm{H}_{15} \mathrm{~N}_{3}$; C, 82.22; H, 4.70; N, 13.08. Found: C, 82.20; H, 4.73; N, 13.04 .

$\mathrm{N}-(($ H-indolo[1,2-c]quinazolin-12-yl)methylene)-2-chlorobenzenamine (4a2)

Compound 3a (1g) was refluxed with 2-Chloroaniline (0.51 g).

(Yield: 1.18 g, 82\%); M.p. $256-257^{\circ} \mathrm{C}$;

FT-IR (vmax , $\left.\mathrm{cm}^{-1}\right) 3055$ (Aromatic C-H str.), 2804 (Aliphatic C-H str.), 1602 (C=N) str.), 1570,1525 (Aromatic C=C ring str.), 1365 (Aromatic C-N str.), 750 (C-Cl str.);

${ }^{1} \mathrm{H}$ NMR (300 MHz, DMSO-d6) $\delta$ ppm: 7.00 (dd, 1H), 7.10 (dd, 2H), 7.20 (dd, 2H), 7.30 (d, 1H), 7.40 (d, 1H), 7.50 (s, $1 \mathrm{H}), 7.58(\mathrm{dd}, 1 \mathrm{H}), 7.60(\mathrm{~d}, 1 \mathrm{H}), 7.83(\mathrm{dd}, 1 \mathrm{H}), 7.84(\mathrm{~d}, 1 \mathrm{H}), 8.01(\mathrm{~d}, 1 \mathrm{H}), 9.23(\mathrm{~s}, 1 \mathrm{H}) ;{ }^{13} \mathrm{C}$ NMR $(75 \mathrm{MHz}, \mathrm{DMSO}-d 6)$

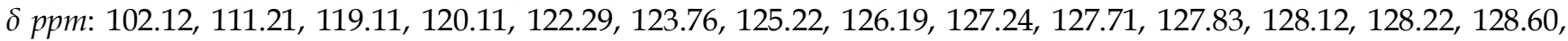
130.24, 134.02, 136.52, 139.22, 144.40, 149.25, 155.35, 160.19; EIMS (m/z): [M]+ 355.35, [M+1] $]^{+} 356.37,[\mathrm{M}+2]^{+}$357.13; Fragments: 217.20, 189.37, 163.24, 138.23, 109.25, 27.15; Anal. calcd. for $\mathrm{C}_{22} \mathrm{H}_{14} \mathrm{~N}_{3} \mathrm{Cl}$; C, 74.26; H, 3.97; $\mathrm{N}, 11.81$. Found: C, 74.22; H, 3.95; N, 11.84 .

$\mathrm{N}-(($ H-indolo[1,2-c]quinazolin-12-yl)methylene)-3-chlorobenzenamine (4a3)

Compound 3a (1g) was refluxed with 3-Chloroaniline (0.51 g).

(Yield: 1.18 g, 82 \%); M.p. $254-255^{\circ} \mathrm{C}$;

FT-IR ( $\left.v_{\max }, \mathrm{cm}^{-1}\right) 3056$ (Aromatic C-H str.), 2929 (Aliphatic C-H str.), 1579 (C=N str.), 1500, 1458 (Aromatic C=C ring str.), 1326 (Aromatic C-N str.), 752 (C-Cl str.);

${ }^{1} \mathrm{H}$ NMR (300 MHz, DMSO-d6) $\delta$ ppm: 7.00 (dd, 1H), 7.10 (dd, 2H), 7.20 (dd, 1H), $7.30(\mathrm{t}, 2 \mathrm{H}), 7.40(\mathrm{~d}, 1 \mathrm{H}), 7.50(\mathrm{~s}$, $1 \mathrm{H}), 7.58(\mathrm{dd}, 1 \mathrm{H}), 7.60(\mathrm{~d}, 1 \mathrm{H}), 7.83(\mathrm{dd}, 1 \mathrm{H}), 7.84(\mathrm{~d}, 1 \mathrm{H}), 8.01(\mathrm{~d}, 1 \mathrm{H}), 9.23(\mathrm{~s}, 1 \mathrm{H})$;

${ }^{13} \mathrm{C}$ NMR (75 MHz, DMSO-d6) $\delta$ ppm: 102.00, 111.24, 119.12, 120.12, 120.45, 122.21, 122.59, 125.22, 126.19, 127.32, $127.41,127.82,128.51,131.43,134.11,135.42,136.71,144.34,149.41,150.45,155.14,160.18$;

EIMS (m/z): [M]+ 355.35, [M+1]+ 356.37, [M+2]+ 357.13; Fragments: 217.20, 189.37, 163.24, 138.23, 109.25, 27.15; Anal. calcd. for $\mathrm{C}_{22} \mathrm{H}_{14} \mathrm{~N}_{3} \mathrm{Cl} ; \mathrm{C}, 74.26 ; \mathrm{H}, 3.97 ; \mathrm{N}, 11.81$. Found: C, 74.22; $\mathrm{H}, 3.95 ; \mathrm{N}, 11.82$.

$\mathrm{N}$-((H-indolo [1,2-c]quinazolin-12-yl)methylene)-4-chlorobenzenamine (4a4)

Compound 3a (1g) was refluxed with 4-Chloroaniline (0.51 g).

(Yield: 1.22 g, $85 \%$ ); M.p. $250-251^{\circ} \mathrm{C}$;

FT-IR $\left(v_{\max }, \mathrm{cm}^{-1}\right) 3041$ (Aromatic C-H str.), 2894 (Aliphatic C-H str.), 1630, 1446 (Aromatic C=C ring str.), 1574 (C=N str.), 1350 (Aromatic C-N str.), 748 (C-Cl str.);

${ }^{1} \mathrm{H}$ NMR $(300 \mathrm{MHz}$, DMSO-d6) $\delta$ ppm: $7.00(\mathrm{dd}, 1 \mathrm{H}), 7.10(\mathrm{dd}, 1 \mathrm{H}), 7.20(\mathrm{dd}, 2 \mathrm{H}), 7.30(\mathrm{dd}, 2 \mathrm{H}), 7.40(\mathrm{~d}, 1 \mathrm{H}), 7.50$ $(\mathrm{s}, 1 \mathrm{H}), 7.58(\mathrm{dd}, 1 \mathrm{H}), 7.60(\mathrm{~d}, 1 \mathrm{H}), 7.83(\mathrm{dd}, 1 \mathrm{H}), 7.84(\mathrm{~d}, 1 \mathrm{H}), 8.01(\mathrm{~d}, 1 \mathrm{H}), 9.23(\mathrm{~s}, 1 \mathrm{H})$;

${ }^{13} \mathrm{C}$ NMR (75 MHz, DMSO-d6) $\delta$ ppm: 102.01, 111.12, 119.01, 120.18, 122.23, 123.71, 123.79, 125.20, 126.14, 127.42, $127.88,128.60,130.21,130.30,132.11,134.09,136.77,144.47,147.35,149.42,155.61,160.14$;

EIMS (m/z): $[\mathrm{M}]+355.35,[\mathrm{M}+1]+356.37,[\mathrm{M}+2]+357.13$; Fragments: 217.20, 189.33, 163.23, 138.23, 109.75, 27.17; Anal. calcd. for $\mathrm{C}_{22} \mathrm{H}_{14} \mathrm{~N}_{3} \mathrm{Cl} ; \mathrm{C}, 74.26 ; \mathrm{H}, 3.97 ; \mathrm{N}, 11.81$. Found: $\mathrm{C}, 74.25 ; \mathrm{H}, 3.95 ; \mathrm{N}, 11.84$.

$\mathrm{N-}(($ H-indolo [1,2-c]quinazolin-12-yl)methylene)-2-nitrobenzenamine (4a5)

Compound $3 \mathrm{a}(\mathrm{gg})$ was refluxed with 2-Nitroaniline $(0.56 \mathrm{~g})$.

(Yield: 1.18 g, 80 \%); M.p. $265-266^{\circ} \mathrm{C}$;

FT-IR ( $\left.v_{\max }, \mathrm{cm}^{-1}\right) 3037$ (Aromatic C-H str.), 2935 (Aliphatic C-H str.), 1611 (C=N str.), 1585,1415 (Aromatic C=C

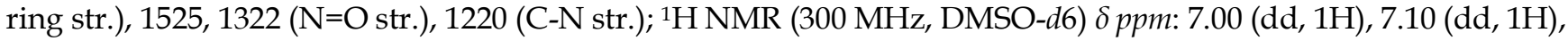


$7.40(\mathrm{~d}, 1 \mathrm{H}), 7.50(\mathrm{~m}, 3 \mathrm{H}), 7.58(\mathrm{dd}, 1 \mathrm{H}), 7.60(\mathrm{~d}, 1 \mathrm{H}), 7.70(\mathrm{dd}, 1 \mathrm{H}), 7.83(\mathrm{dd}, 1 \mathrm{H}), 7.84(\mathrm{~d}, 1 \mathrm{H}), 8.01(\mathrm{~d}, 1 \mathrm{H}), 8.20(\mathrm{~d}$, $1 \mathrm{H}), 9.23(\mathrm{~s}, 1 \mathrm{H})$;

${ }^{13} \mathrm{C}$ NMR (75 MHz, DMSO-d6) $\delta$ ppm: 102.05, 111.10, 119.04, 120.12, 122.25, 122.45, 123.29, 125.28, 126.12, 127.45, 127.86, 128.20, 128.61, 134.12, 136.23, 136.71, 141.92, 144.13, 144.56, 149.40, 155.93, 160.12;

EIMS (m/z): [M]+ 366.76, [M+1]+ 367.36; Fragments: 217.16, 189.37, 163.44, 149.73, 109.25, 27.10; Anal. calcd. for $\mathrm{C}_{22} \mathrm{H}_{14} \mathrm{~N}_{4} \mathrm{O}_{2} ; \mathrm{C}, 72.12 ; \mathrm{H}, 3.85 ; \mathrm{N}, 15.29$. Found: $\mathrm{C}, 72.10 ; \mathrm{H}, 3.82 ; \mathrm{N}, 15.33$.

$\mathrm{N}-(($ H-indolo [1,2-c]quinazolin-12-yl)methylene)-3-nitrobenzenamine (4a6)

Compound 3a $(1 \mathrm{~g})$ was refluxed with 3-Nitroaniline $(0.56 \mathrm{~g})$.

(Yield: 1.19 g, $81 \%$ ); M.p. $264-265^{\circ} \mathrm{C}$;

FT-IR ( $\left.v_{\max }, \mathrm{cm}^{-1}\right) 3051$ (Aromatic C-H str.), 2935 (Aliphatic C-H str.), 1635 (C=N str.), 1585,1419 (Aromatic C=C ring str.), 1508, 1360 (N=O str.), 1174 (C-N str.); ${ }^{1} \mathrm{H}$ NMR (300 MHz, DMSO-d6) $\delta p p m: 7.00$ (dd, 1H), 7.10 (dd, 1H), $7.40(\mathrm{~d}, 1 \mathrm{H}), 7.50(\mathrm{~m}, 2 \mathrm{H}), 7.58(\mathrm{dd}, 1 \mathrm{H}), 7.60(\mathrm{~d}, 1 \mathrm{H}), 7.70(\mathrm{~d}, 1 \mathrm{H}), 7.83(\mathrm{dd}, 1 \mathrm{H}), 7.84(\mathrm{~d}, 1 \mathrm{H}), 8.01(\mathrm{~d}, 1 \mathrm{H}), 8.20(\mathrm{~m}$, $2 \mathrm{H}), 9.23(\mathrm{~s}, 1 \mathrm{H})$;

${ }^{13} \mathrm{C}$ NMR (75 MHz, DMSO-d6) $\delta$ ppm: 102.12, 111.16, 117.11, 119.27, 119.61, 120.71, 122.77, 125.23, 126.18, 127.42, $127.83,128.44,128.64,131.03,134.39,136.54,144.67,149.42,149.56,149.92,155.90,160.12$;

EIMS (m/z): [M]+ 366.36, [M+1]+ 367.39; Fragments: 217.16, 189.36, 163.17, 149.33, 109.27, 27.10; Anal. calcd. for $\mathrm{C}_{22} \mathrm{H}_{14} \mathrm{~N}_{4} \mathrm{O}_{2} ; \mathrm{C}, 72.12 ; \mathrm{H}, 3.85 ; \mathrm{N}, 15.29$. Found: C, 72.09; H, 3.81; N, 15.32 .

$\mathrm{N-}(($ H-indolo[1,2-c]quinazolin-12-yl)methylene)-4-nitrobenzenamine (4a7)

Compound 3a $(1 \mathrm{~g})$ was refluxed with 4-Nitroaniline (0.56 g).

(Yield: 1.19 g, 81 \%); M.p. $270-271^{\circ} \mathrm{C}$;

FT-IR ( $\left.v_{\max }, \mathrm{cm}^{-1}\right) 3080$ (Aromatic C-H str.), 2958 (Aliphatic C-H str.), 1685 (C=N str.), 1602,1421 (Aromatic C=C ring str.), 1554, 1326 (N=O str.), 1292 (C-N str.); ${ }^{1} \mathrm{H}$ NMR (300 MHz, DMSO-d6) $\delta p p m: ~ 7.00$ (dd, 1H), 7.10 (dd, 1H), $7.40(\mathrm{~d}, 1 \mathrm{H}), 7.50(\mathrm{~m}, 2 \mathrm{H}), 7.58(\mathrm{dd}, 1 \mathrm{H}), 7.60(\mathrm{~d}, 1 \mathrm{H}), 7.70(\mathrm{~d}, 1 \mathrm{H}), 7.83(\mathrm{dd}, 1 \mathrm{H}), 7.84(\mathrm{~d}, 1 \mathrm{H}), 8.01(\mathrm{~d}, 1 \mathrm{H}), 8.20(\mathrm{dd}$, $2 \mathrm{H}), 9.23(\mathrm{~s}, 1 \mathrm{H})$;

${ }^{13} \mathrm{C}$ NMR (75 MHz, DMSO-d6) $\delta$ ppm: 102.11, 111.12, 119.04, 120.13, 122.20, 122.44, 122.79, 123.20, 123.24, 125.22, $126.18,127.40,127.81,128.62,134.01,136.79,144.47,146.92,149.45,155.12,155.91,160.13$;

EIMS (m/z): [M]+ 366.76, [M+1]+ 367.16; Fragments: 217.13, 189.37, 163.47, 149.27, 109.64, 27.14; Anal. calcd. for $\mathrm{C}_{22} \mathrm{H}_{14} \mathrm{~N}_{4} \mathrm{O}_{2} ; \mathrm{C}, 72.12 ; \mathrm{H}, 3.85 ; \mathrm{N}, 15.29$. Found: $\mathrm{C}, 72.11 ; \mathrm{H}, 3.87 ; \mathrm{N}, 15.28$.

$\mathrm{N-}(($ H-indolo[1,2-c]quinazolin-12-yl)methylene)-4-fluorobenzenamine (4a8)

Compound $3 \mathrm{a}(\mathrm{g})$ was refluxed with 4-Fluoroaniline $(0.44 \mathrm{~g})$.

(Yield: 1.17 g, $86 \%$ ); M.p. $245-246^{\circ} \mathrm{C}$;

FT-IR ( $\left.v_{\max }, \mathrm{cm}^{-1}\right) 3075$ (Aromatic C-H str.), 2925 (Aliphatic C-H str.), 1621, 1454 (Aromatic C=C ring str.), 1555 (C=N str.), 1325 (Aromatic C-N str.), 1292 (C-F str.); ${ }^{1} \mathrm{H}$ NMR (300 MHz, DMSO-d6) $\delta$ ppm: 7.00 (dd, 3H), 7.10 (dd, $1 \mathrm{H}), 7.20(\mathrm{dd}, 2 \mathrm{H}), 7.40(\mathrm{~d}, 1 \mathrm{H}), 7.50(\mathrm{~s}, 1 \mathrm{H}), 7.58(\mathrm{dd}, 1 \mathrm{H}), 7.60(\mathrm{~d}, 1 \mathrm{H}), 7.83(\mathrm{dd}, 1 \mathrm{H}), 7.84(\mathrm{~d}, 1 \mathrm{H}), 8.01(\mathrm{~d}, 1 \mathrm{H}), 9.23$ $(\mathrm{s}, 1 \mathrm{H})$;

${ }^{13} \mathrm{C}$ NMR (75 MHz, DMSO-d6) $\delta$ ppm: 102.02, 111.15, 116.81, 116.84, 119.03, 120.21, 122.29, 123.24, 123.48, 125.25, $126.18,127.42,127.81,128.65,134.02,136.71,144.49,144.67,149.49,155.91,160.12,161.4$;

EIMS (m/z): [M] 339.39, [M+1] $]^{+}$340.07; Fragments: 217.27, 189.24, 163.42, 122.24, 109.49, 27.14; Anal. calcd. for $\mathrm{C}_{22} \mathrm{H}_{14} \mathrm{~N}_{3} \mathrm{~F} ; \mathrm{C}, 77.86 ; \mathrm{H}, 4.16 ; \mathrm{N}, 12.38$. Found: $\mathrm{C}, 77.84 ; \mathrm{H}, 4.19 ; \mathrm{N}, 12.35$.

$\mathrm{N}-(($ H-indolo[1,2-c]quinazolin-12-yl)methylene)-4-bromobenzenamine (4a9)

Compound 3a $(1 \mathrm{~g})$ was refluxed with 4-Bromoaniline $(0.69 \mathrm{~g})$.

(Yield: 1.37 g, 85 \%); M.p. $245-246^{\circ} \mathrm{C}$;

FT-IR ( $\left.v_{\max }, \mathrm{cm}^{-1}\right) 3051$ (Aromatic C-H str.), 2940 (Aliphatic C-H str.), 1609,1458 (Aromatic C=C ring str.), 1573 (C=N str.), 1327 (Aromatic C-N str.), 551 (C-Br str.); ${ }^{1} \mathrm{H}$ NMR (300 MHz, DMSO-d6) $\delta$ ppm: 7.00 (dd, 1H), 7.20 (dd, $2 \mathrm{H}), 7.30(\mathrm{~d}, 1 \mathrm{H}), 7.40(\mathrm{dd}, 3 \mathrm{H}), 7.50(\mathrm{~s}, 1 \mathrm{H}), 7.58(\mathrm{dd}, 1 \mathrm{H}), 7.60(\mathrm{~d}, 1 \mathrm{H}), 7.83(\mathrm{dd}, 1 \mathrm{H}), 7.84(\mathrm{~d}, 1 \mathrm{H}), 8.01(\mathrm{~d}, 1 \mathrm{H}), 9.23$ $(\mathrm{s}, 1 \mathrm{H})$; 
${ }^{13} \mathrm{C}$ NMR (75 MHz, DMSO-d6) $\delta$ ppm: 102.02, 111.14, 119.05, 120.14, 121.63, 122.22, 124.59, 124.67, 125.18, 126.42, $127.12,127.81,128.62,133.03,133.41,134.08,136.77,144.14,148.41,149.23,155.71,160.14$;

EIMS (m/z): [M]+ 399.24, [M+1]+ 400.39, [M+2]+ 401.62; Fragments: 217.27, 189.22, 181.24, 163.42, 109.27, 27.99; Anal. calcd. for $\mathrm{C}_{22} \mathrm{H}_{14} \mathrm{~N}_{3} \mathrm{Br} ; \mathrm{C}, 77.86 ; \mathrm{H}, 4.16 ; \mathrm{N}, 12.38$. Found: $\mathrm{C}, 77.85 ; \mathrm{H}, 4.15 ; \mathrm{N}, 12.39$.

$N-((H-i n d o l o[1,2-c] q u i n a z o l i n-12-y l) m e t h y l e n e)-2-c h l o r o-4-n i t r o b e n z e n a m i n e(4 a 10)$

Compound 3a $(1 \mathrm{~g})$ was refluxed with 2-Chloro-4-nitroaniline $(0.69 \mathrm{~g})$.

(Yield: 1.29 g, $80 \%$ ); M.p. $258-259^{\circ} \mathrm{C}$;

FT-IR ( $\left.v_{\max }, \mathrm{cm}^{-1}\right) 3037$ (Aromatic C-H str.), 2920 (Aliphatic C-H str.), 1609 (C=N str.), 1556, 1423 (Aromatic C=C ring str.), 1513,1308 (N=O str.), 1283 (C-N str.), 692 (C-Cl str.); ${ }^{1} \mathrm{H}$ NMR (300 MHz, DMSO-d6) $\delta$ ppm: 7.00 (dd, $1 \mathrm{H}), 7.10(\mathrm{dd}, 1 \mathrm{H}), 7.40(\mathrm{~d}, 1 \mathrm{H}), 7.50(\mathrm{~m}, 2 \mathrm{H}), 7.58(\mathrm{dd}, 1 \mathrm{H}), 7.60(\mathrm{~d}, 1 \mathrm{H}), 7.83(\mathrm{dd}, 1 \mathrm{H}), 7.84(\mathrm{~d}, 1 \mathrm{H}), 8.01(\mathrm{~d}, 1 \mathrm{H})$, $8.10(\mathrm{~d}, 1 \mathrm{H}), 8.20(\mathrm{~s}, 1 \mathrm{H}), 9.23(\mathrm{~s}, 1 \mathrm{H})$;

${ }^{13} \mathrm{C}$ NMR (75 MHz, DMSO-d6) $\delta$ ppm: 102.04, 111.14, 119.03, 120.12, 120.56, 122.22, 124.61, 125.29, 125.36, 126.14, $127.45,127.85,128.63,128.72,134.01,136.74,144.45,145.28,148.36,149.47,155.91,160.16$;

EIMS (m/z): [M] ${ }^{+}$400.31, [M+1]+ 401.17, [M+2]+ 402.18; Fragments: 217.27, 189.01, 181.24, 163.73, 109.26, 27.96; Anal. calcd. for $\mathrm{C}_{22} \mathrm{H}_{13} \mathrm{~N}_{4} \mathrm{O}_{2} \mathrm{Cl} ; \mathrm{C}, 65.92 ; \mathrm{H}, 3.27 ; \mathrm{N}, 13.98$. Found: $\mathrm{C}, 65.88 ; \mathrm{H}, 3.30 ; \mathrm{N}, 13.99$.

N-((H-indolo[1,2-c]quinazolin-12-yl)methylene)-4-chloro-2-nitrobenzenamine(4a11)

Compound 3a $(1 \mathrm{~g})$ was refluxed with 4-Chloro-2-nitroaniline $(0.69 \mathrm{~g})$.

(Yield: 1.29 g, $80 \%$; M.p. $260-261^{\circ} \mathrm{C}$ )

FT-IR ( $\left.v_{\max }, \mathrm{cm}^{-1}\right) 3019$ (Aromatic C-H str.), 2942 (Aliphatic C-H str.), 1617 (C=N str.), 1570, 1446 (Aromatic C=C ring str.), 1520, 1330 (N=O str.), 1283 (C-N str.), 702 (C-Cl str.); ${ }^{1} \mathrm{H}$ NMR (300 MHz, DMSO-d6) $\delta$ ppm: 7.00 (dd, 1H), $7.10(\mathrm{dd}, 1 \mathrm{H}), 7.40(\mathrm{~d}, 1 \mathrm{H}), 7.50(\mathrm{~m}, 2 \mathrm{H}), 7.58(\mathrm{dd}, 1 \mathrm{H}), 7.60(\mathrm{~s}, 1 \mathrm{H}), 7.70(\mathrm{~d}, 1 \mathrm{H}) 7.83(\mathrm{dd}, 1 \mathrm{H}), 7.84(\mathrm{~d}, 1 \mathrm{H}), 8.01(\mathrm{~d}$, $1 \mathrm{H}), 8.20(\mathrm{~s}, 1 \mathrm{H}), 9.23(\mathrm{~s}, 1 \mathrm{H})$;

${ }^{13} \mathrm{C}$ NMR (75 MHz, DMSO-d6) $\delta$ ppm: 102.05, 111.14, 119.23, 120.14, 122.25, 124.66, 125.25, 125.36, 126.17, 127.46, $127.81,128.62,133.77,134.01,136.34,136.71,142.29,143.37,144.45,149.40,155.92,160.14$;

EIMS (m/z): [M] $]^{+}$400.31, [M+1] $]^{+}$401.17, [M+2]+ 402.18; Fragments: 217.27, 189.21, 181.24, 163.73, 109.70, 27.02; Anal. calcd. for $\mathrm{C}_{22} \mathrm{H}_{13} \mathrm{~N}_{4} \mathrm{O}_{2} \mathrm{Cl} ; \mathrm{C}, 65.92 ; \mathrm{H}, 3.27 ; \mathrm{N}, 13.98$. Found: $\mathrm{C}, 65.89 ; \mathrm{H}, 3.31 ; \mathrm{N}, 13.95$.

\subsection{Antibacterial activity}

The antibacterial potential of all compounds was tested and compared with ciprofloxacin using agar well diffusion method. Activity was performed against gram negative bacteria Escherichia coli and gram positive bacteria Bacillus subtilis. Test microorganisms were obtained from Microbiology Laboratory, National JALMA Institute for Leprosy \& Other Mycobacterial Diseases, Agra, India. Synthesized compounds were not shown significant antibacterial activity at the concentration range of 10 to $30 \mu \mathrm{g} / \mathrm{ml}$. So, the higher concentrations of compounds were used to evaluate antibacterial efficacy. Solution with concentration of $100 \mu \mathrm{g} / \mathrm{ml}$ and $150 \mu \mathrm{g} / \mathrm{ml}$ of each test compound was prepared in Dimethylsulfoxide (DMSO). All materials used were sterilized. The microorganisms were grown in nutrient broth at $37^{\circ} \mathrm{C}$ for $24 \mathrm{~h}$. Nutrient agar was melted on a water bath and cooled to $45^{\circ} \mathrm{C}$. Then it was shaked gently for uniform cooling. Each culture $(1.0 \mathrm{ml})$ was inoculated aseptically and then it was mixed by gentle shaking and then poured into the already sterilized petridishes. Then the material was allowed to settle for 1-2 $\mathrm{h}$ and then solid medium was cut to make wells of $7 \mathrm{~mm}$. A solution of each compound was added to each of these wells. Then these plates were incubated at $37^{\circ} \mathrm{C}$ for $24 \mathrm{~h}$. After the time of incubation, zone of inhibition around each of these wells were measured in millimeters. DMSO was used as a control. Ciprofloxacin was used as a reference standard [36].

\subsection{Antioxidant activity}

\subsubsection{DPPH radical scavenging assay}

This assay is a standard method for the evaluation of antioxidant activity of compounds. Free radical scavenging ability of synthesized compounds was compared with ascorbic acid, which is a standard antioxidant. To evaluate antioxidant activity of compounds, $0.1 \mathrm{mM}$ solution of DPPH was prepared by dissolving $1.9 \mathrm{mg}$ 
DPPH in methanol. To form the free radicals, solutions were kept in darkness for half an hour. Sample solutions of different concentrations were prepared $(10,20,30,40$ and $50 \mu \mathrm{g} / \mathrm{ml})$. Solutions of same concentrations were prepared with ascorbic acid and they were used as standard. $1 \mathrm{ml}$ of solution of each test compound was added with same volume of DPPH solution, mixed vigorously and then kept in dark room for half an hour. Absorbance of all solutions was measured at $517 \mathrm{~nm}$. Procedure was performed in triplicate manner $(\mathrm{n}=3)$ and the average of three readings with standard deviation was shown in results. Same procedure was done with the ascorbic acid solutions. Percent scavenging was calculated using equation 1 [37].

$\%$ inhibition $=\frac{\text { Absorbance of control-Absorbance of sample }}{\text { Absorbance of control }} \times 100$

\subsection{2. $\mathrm{H}_{2} \mathrm{O}_{2}$ radical scavenging activity}

In this study hydrogen peroxide was used to form hydroxyl radicals. Standard and sample solutions were prepared as above and concentrations of hydroxyl radicals were evaluated at $230 \mathrm{~nm}$ [38]. Procedure was performed in triplicate manner and the average of three readings with standard deviation was shown in results. Percent inhibition (or \% scavenging) activity was calculated by using equation 1.

Acknowledgements: Authors would like to thank management of Rajiv Academy for Pharmacy, Mathura for providing research facilities. IIT, Delhi is acknowledged for providing the spectral data of the synthesized compounds. National JALMA Institute for Leprosy \& Other Mycobacterial Diseases, Agra is acknowledged for providing bacterial cultures.

Author contributions: Concept -A.D., D.P., G.K.S.; Design - A.D., D.P., G.K.S.; Supervision -G.K.S. Resource - A.D., D.P., G.K.S.; Materials - A.D., D.P., G.K.S.; Data Collection and/or Processing -A.D.; Analysis and/or Interpretation A.D.; Literature Search -A.D.; Writing -A.D.; Critical Reviews A.D., D.P., G.K.S.

Conflict of Interest: Authors have no conflict of interest.

\section{REFERENCES}

[1] Somei M, Yamada F. Simple indole alkaloids and those with a nonrearranged monoterpenoid unit. Nat Prod Rep. 2003; 20: 216-242. [CrossRef]

[2] Gupta L, Talwar A, Chauhan PMS. Bis and tris indole alkaloids from marine organisms: new leads for drug discovery. Curr Med Chem. 2007; 14: 1789-1803. [CrossRef]

[3] Xu H, Lv M. Developments of indoles as anti-HIV-1 inhibitors. Curr Pharm Des. 2009; 15: 2120-2148. [CrossRef]

[4] Ran J, Huang N, Xu H, Yang Y, Lv M, Zheng YT. Anti HIV-1 agents 5: Synthesis and anti-HIV-1 activity of some Narylsulfonyl-3-acetylindoles. Bioorg Med Chem Lett. 2010; 20: 3534-3536. [CrossRef]

[5] Ghosh AK, Gong G, Grum-Tokars V, Mulhearn DC, Baker SC, Coughlin M, Prabhakar BS, Sleeman K, Johnson ME, Mesecar AD. Design, synthesis and antiviral efficacy of a series of potent chloropyridyl ester-derived SARS-CoV 3CLpro inhibitors. Bioorg Med Chem Lett. 2008; 18: 5684-5688. [CrossRef]

[6] Williams JD, Chen JJ, Drach JC, Townsend LB. Synthesis and antiviral activity of 3-formyl- and 3-Cyano-2,5,6trichloroindole nucleoside derivatives. J Med Chem. 2004; 47: 5766-5772. [CrossRef]

[7] Andreani A, Burnelli S, Granaiola M, Leoni A, Locatelli A, Morigi R, Rambaldi M, Varoli L, Landi L, Prata C, Berridge MV, Grasso C, Fiebig HH, Kelter G, Burger AM, Kunkel MW. Antitumor activity of bis-indole derivatives. J Med Chem. 2008; 51: 4563-4570. [CrossRef]

[8] Slater MJ, Baxter R, Bonser RW, Cockerill S, Gohil K, Parry N, Robinson E, Randall R, Yeates C, Snowden W, Walters A. Synthesis of $\mathrm{N}$-alkyl substituted indolocarbazoles as potent inhibitors of human cytomegalovirus replication. Bioorg Med Chem Lett. 2001; 11: 1993-1995. [CrossRef] 
[9] Mathada BSD, Mathada MBH. Synthesis and antimicrobial activity of some 5-substituted-3-phenyl-Nb-(Substituted-2oxo-2H-pyrano [2,3- $b$ ]quinoline-3-carbonyl)-1H-indole-2-carboxyhydrazide. Chem Pharm Bull. 2009; 57: 557-560. [CrossRef]

[10] Gurkok G, Altanlar N, Suzen S. Investigation of antimicrobial activities of indole-3-aldehyde hydrazide/hydrazone derivatives. Chemotherapy. 2009; 55, 15-19. [CrossRef]

[11] Karah N, Gursoy A, Kandemirli F, Shvets N, Kaynak FB, Ozbey S, Kovalishyn V, Dimoglo A. Synthesis and structureantituberculosis activity relationship of $1 \mathrm{H}$-indole-2,3-dione derivatives. Bioorg Med Chem. 2007; 15: 5888-5891. [CrossRef]

[12] Dekker WH, Selling HA, Overeem JC. Structure-activity relationships of some antifungal indoles. J Agric Food Chem. 1975; 23: 785-791. [CrossRef]

[13] Whitehead CW, Whitesitt AA. Effects of lipophilic substituents on some biological properties of indoles. J Med Chem. 1974; 17: 1298-1304. [CrossRef]

[14] Malesani G, Chiarelotto G, Dallacqua F, Vedali D. Antimicrobial properties of some 3-acyl-4,7- disubstituted indoles. Farmaco. 1975; 30: 137-146.

[15] Donawade DS, Gadaginamath GS. Some electrophilic substitution reactions on 1-substituted-3-acetyl/carbethoxy-5hydroxy-2-methylindole and antimicrobial activity of these new indole derivatives. Indian J Chem. 2005; 44B: 1679-1685.

[16] Tsotinis A, Varvaresou A, Calogeropoulou T, Siatra-Papastaikoudi T, Tiligada A. Synthesis and antimicrobial evaluation of indole containing derivatives of 1,3,4-triazole and their open-chain counterparts. Drug Res. 1997; 47: $307-310$.

[17] Varvaresou A, Tsantili-Kakoulidou A, Siatra-Papastaikoudi T, Tiligada E. Synthesis and biological evaluation of indole containing derivatives of thiosemicarbazide and their cyclic 1,2,4-triazole and 1,3,4-thiadiazole analogs. Drug Res. 2000; 50: 48-54. [CrossRef]

[18] Macchia M, Manera C, Nencetti S, Rossello A, Broccali G, Limonta D. Synthesis and antimicrobial activity of benzo[ $a]$ dihydrocarbazole and benzotetrahydrocyclohept $[1,2 b]$ indole derivatives. Farmaco. 1996; 51: 75-78.

[19] Gadaginamath GS, Kavali RR. Synthesis and antimicrobial activity of novel 4H-pyrano [2, 3-f]- indole derivatives. Indian J Chem. 1999; 38B: 178-182.

[20] Bhovi MG, Gadaginamath GS. 1, 3-Dipolarcycloaddition reactions: Synthesis and antimicrobial activity of novel 1triazolylindole and 1-triazolylbenz-[g]indole derivatives. Indian J Chem. 2005; 44B: 1068-1073.

[21] Bast A, Haenen G, Doelman C. Oxidants and antioxidants: state of the art. Am J Med. 1991; 92 (Suppl. 3C): 2-13. [CrossRef]

[22] Bulkley GB. Free radicals and other reactive oxygen metabolites: Clinical relevance and the therapeutic efficacy of antioxidant therapy. Surgery. 1993; 113: 479-483.

[23] Halliwell B, Gutteridge M. Free radicals in biology and medicine, third ed. Oxford Science Publications. Oxford University Press. 1998.

[24] Sreejayan N, Rao MN. Free radical scavenging activity of curcuminoids. Drug Res. 1996; 46: 169-171.

[25] McCord JM. Human disease, free radicals, and the oxidant/antioxidant balance. Clin Biochem. 1993; $26: 351-357$. [CrossRef]

[26] Cross CE, Vliet A, Neill ACO, Eiserich JP. Reactive oxygen species and the lung. Lancet. 1994; 344: 930-933. [CrossRef]

[27] Santrucek M, Krepelka J. Antioxidants from the aspect of their potential use in chemotherapy. Drugs Future. 1988; 37: 121-128. [CrossRef]

[28] Santrucek M, Krepelka J. Of particular interests are the potential protective effects of antioxidants on lipoproteins, since oxidized LDL is thought to be atherogenic. Drugs Future. 1988; 13: 973-996.

[29] Nikolic D, Breemen RB. DNA Oxidation Induced by Cyclooxygenase-2. Chem Res Toxicol. 2001; 14: 351-354. [CrossRef]

[30] Vapaatalo H. Free radicals and anti-inflammatory drugs. Med Biol. 1986; 64: 1-7.

[31] Halliwell B, Hoult JR, Blake DR. Oxidants, inflammation, and anti-inflammatory drugs. FASEB J. $1988 ; 2: 2867-2873$. [CrossRef] 
[32] Mouithys-Mickalad AML, Zheng SX, Deby-Dupont GP, Deby CM, Lamy MM, Reginster JY, Henrotin YE. In vitro study of the antioxidant properties of nonsteroidal anti-inflammatory drugs by chemiluminescence and electron spin resonance (ESR). Free Radic Res. 2000; 33: 607-621. [CrossRef]

[33] Turrens JF. Mitochondrial formation of reactive oxygen species. J Physiol. 2003; 552: 335-344. [CrossRef]

[34] Dedon PC, Tannenbaum SR. Reactive nitrogen species in the chemical biology of inflammation. Arch Biochem Biophys. 2004; 423: 12-22. [CrossRef]

[35] Billimoria AD, Cava MP. Chemistry of indolo[1, 2-c]quinazoline: An approach to the marine alkaloid Hinckdentine A. J Org Chem. 1994; 59: 6777-6782. [CrossRef]

[36] Ugur A, Mercimek B, Ozler MA, Aahin N. Antimicrobial effects of bis (2-imidazolinyl)-5,5V-dioxime and its mono- and tri-nuclear complexes. Transit Met Chem. 2000; 25: 421. [CrossRef]

[37] Malviya R, Sharma PK, Dubey SK. Antioxidant potential and emulsifying properties of kheri (Acacia chundra, Mimosaceae) gum polysaccharide. Marm Pharm J. 2017; 21(3): 701-706. [CrossRef]

[38] Ningsih IY, Zulaikhah S, Hidayat MA, Kuswandi B. Antioxidant activity of various Kenitu (Chrysophyllum cainito L.) leaves extracts from Jember. Indonesia Agric Sci Procedia. 2016; 9: 378-385. [CrossRef]

This is an open access article which is publicly available on our journal's website under Institutional Repository at http://dspace.marmara.edu.tr. 